\title{
USING 360-DEGREE INTERACTIVE PANORAMAS TO DEVELOP VIRTUAL REPRESENTATIONS OF CONSTRUCTION SITES
}

\author{
Ricardo Eiris Pereira ${ }^{1}$, Hashem Izadi Moud ${ }^{2}$, and Masoud Gheisari ${ }^{3}$
}

\begin{abstract}
: 360-degree panoramic virtual reality is a ubiquitous technique for visualizing complex construction environments that can be augmented with different layers of information. Unlike the common virtual environments that provide unrealistic computer-generated simulation of the environment, panoramic virtual reality is able to create highly realistic and detailed representations of the real environment while giving users a sense of immersion. In this study, interactive panoramas are made of a series of panoramic videos and photos, which are captured from a construction site. Various types of time/location-specific information (e.g., audio, video, virtual 2D, or 3D models) can be augmented in such panoramic virtual environments to create a natural interactive experience. Users will be able to navigate through the panoramic virtual environment of a complex construction project while interacting with augmented information to learn more about the construction project and job site. In this research a 360-degree panoramic virtual reality environment of a construction job site is created. This paper describes the development of interactive panoramic scenes using panoramic photos and videos. The procedure of creating the panoramic experience, the process of augmenting the captured panoramas with different layers of information and an integrated pilot study are described in details.
\end{abstract}

Keywords: 360-degree panorama, interactive panoramic scenes, virtual reality, immersive virtual experience, construction site visit.

\section{INTRODUCTION}

Complex real world simulations are very computational intensive and time consuming using traditional virtual reality techniques, where computer-generated representations of the environment are modeled from a user's perception of reality. 3D computer graphics allow users to synthesize an environment for arbitrary representations, but the rendering quality and scene complexity are often limited because of the real-time constraints (Vanijja and Horiguchi 2006). On the contrary, 360-degree panoramas are unbroken views of a whole region surrounding an observer, giving a "sense of presence, of being there" (Bourke 2014) to the observer. Panoramic reality capturing techniques create an un-modeled view of the real environment that looks identical to the true reality. This provides inherent benefits over traditional virtual reality techniques. 360-degree panoramas offer low computational, easy to capture, non-computer generated

1 Ph.D. Student, Rinker School of Construction Management, University of Florida, Gainesville, FL, USA, reiris@ufl.edu

2 Ph.D. Student, Rinker School of Construction Management, University of Florida, Gainesville, FL, USA, izadimoud@ufl.edu

3 Assistant Professor, Rinker School of Construction Management, University of Florida, Gainesville, FL, USA, masoud@ufl.edu 
simulations that are immersive to the user, due to the realism embedded in photography and videography data.

Panoramic photos and videos have variety of application in different areas including professional training and virtual tour representation of complex environments (Vanijja and Horiguchi 2001). Interactive panoramic scenes have also been used for several applications by researchers in the construction domain. Early research focused on addressing the technicalities of creation, capturing, interpretation, and navigation of 360degree images and video. Finch and Wing (1996) employed video-still images on a computer-based system to produce a navigable simulator for students in built environment disciplines. Mei and Wing (1999) used a series of interconnected 360degree panoramic images that enabled users to navigate from one image to another through an interface to visit virtual construction sites. Ellis et al. (2004), developed a computer-based learning resource that used overlapping images to explore a virtual panoramic site.

Recent research projects have used 360-degree scenes as a reality backdrop to augment mainly 3D models on them (Côté et al. 2011a, Wither et al. 2011). Côté et al. (2011b) used a panorama of surface of a street to augment a virtual excavation project and illustrated underground utilities on it. Gheisari et al. (2015) applied interactive panoramas as location-independent solution to illustrate free body diagrams of the structural elements of the building to students. Gheisari et al. (2015) showed that panoramic scenes have the potential to enable any user to experience construction job site environment without being physically present on the job site. Gheisari et al. (2016a; 2016b) also used a 360-degree image of a construction renovation project and superimposed building information models on it to provide construction personnel with a location-independent way to access the required information in an immersive interactive environment. Additionally, emerging software packages (e.g. Agisoft PhotoScan, NCTech ColourCloud, and Arithmetica SphereVision) constantly expands construction applications of interactive panoramas. Some of the new capabilities built on 360-degree panoramas include: project assets documentation and management, virtual tour creation, point-cloud colorization, image-to-point cloud processing, and point-topoint measurements photogrammetry.
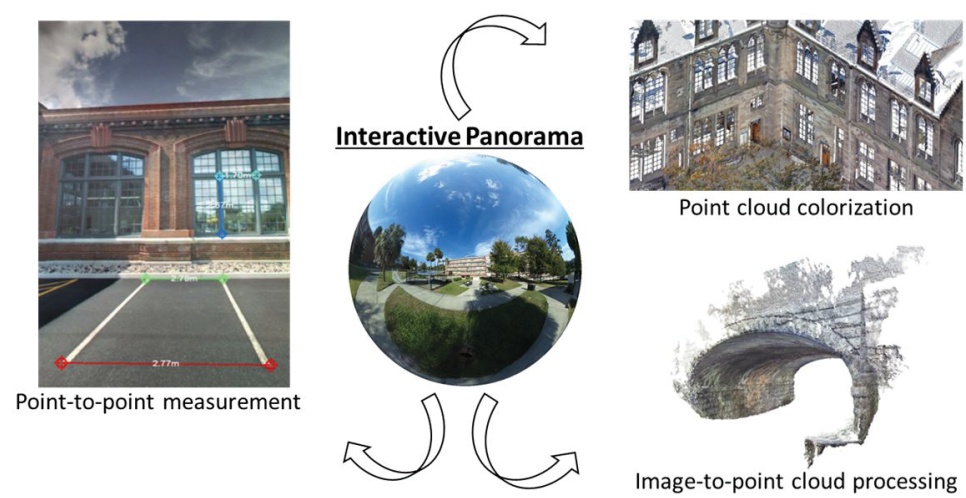

Figure 1. Construction Applications of Interactive Panoramas (Adapted from Perez-Ramos, A. 2016)

This study aims to describe the workflow of creating an interactive panoramic scene using 360-degree photos and videos, hardware and software requirements, and augmentation process of captured panoramas with different layers of information. 
Additionally, a pilot case study is provided where the collection of technologies and techniques are applied in a real construction project.

\section{360-DEgreE INTERACTIVE PANORAMA}

Interactive panoramas employ uninterrupted images and videos augmented with data to provide immersive visualization of the real environments. These 360-degree scenes enhance users' perception of the real environment by introducing different layers of information, such as audio, text, video, 2D and 3D objects. The panoramic photos and videos allow users to maneuver through an environment, without being physically there (Finch and Wing 1996), by presenting an immersive simulation of traversing a particular environment. The user is able to move in a number of predetermined directions and explore the virtual representation of the site.

New technologies have enhanced the process of image and video capturing, taking it from an intricate robotic photographic system (Mei and Wing 1999), to a simple handheld camera or smartphone that acquires pictures of the location in a few seconds. The editing process has been made easy with automated software, requiring minimal effort to create multidirectional scenes with the capability of user movement. The workflow to create a 360-degree interactive panorama is described in the subsequent section.

\subsection{Capturing, Authoring, Immersion, and Distribution}

Figure 2 illustrates the four-stage process for creation of an interactive panorama: Capturing, Authoring, Immersion, and Distribution. This section discusses each stage in details.

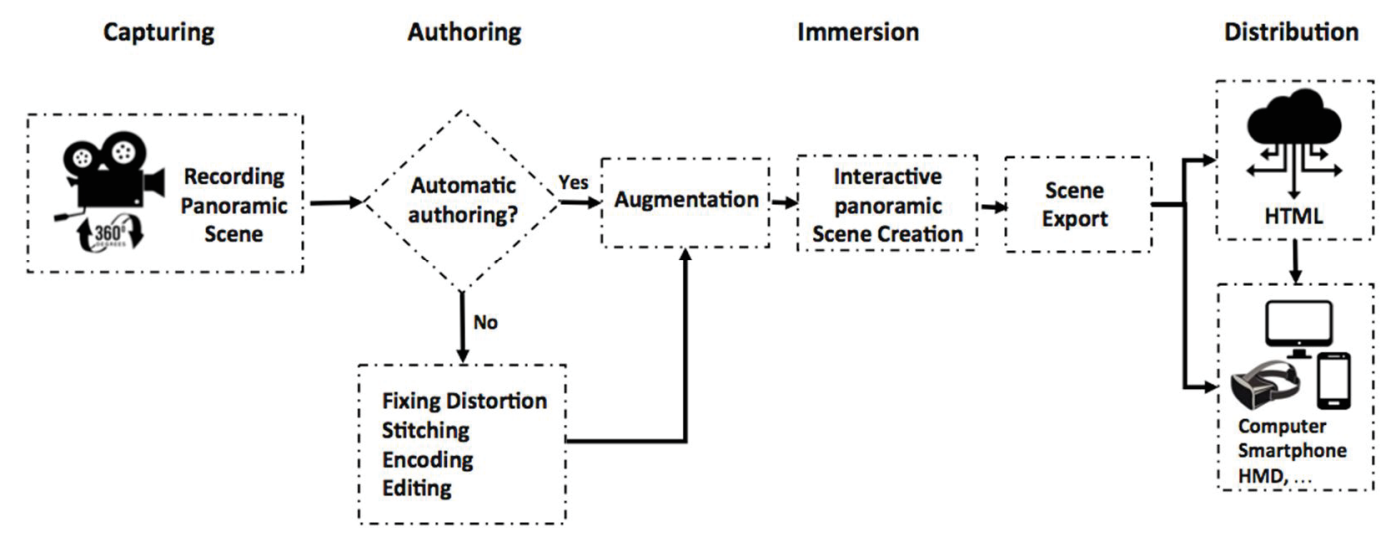

Figure 2. The Workflow of Creating an Interactive Panorama.

The capture of a panoramic scene is obtained with two common methods: (1) using a camera to take spatially located shots from a pivot point, recording with traditional angled lens video and images, or (2) using camera with ultra-wide-angle lenses to document spherical 360-degree video and images (Nayar 1997). The first technique can be achieved by taking multiple rows of photos shots with a single rotating camera, or multiple simultaneous cameras, in a spherical or partial cylindrical fashion (from a static point) to recreate a full 360-degree panorama. The second technique for data capturing employs fish-eye lens or catadioptric lens systems on a stationary location to produce strong visually distorted images due to the wide-angle of the camera. 
The authoring process uses specialized software to adjust the geometric displacement of the surface projection by performing projective geometry transformations. Computer algorithms have been developed to automate the distortion correction tasks (Nayar and Boult 1998, Brauer-Burchardt and Voss 2001), permitting the stitching, encoding and editing the images. Once the geometric displacements are corrected, the interpretation of the corrected graphical projections can be easily done by users to examine it in a 360degree spherical context.

The immersion process introduces layers of information enhancing and augmenting the content presented in the authorised panoramic scene. Additionally, audio layers can be integrated into scenes to increase the immersion of the user by making the panoramic scene a more realistic representation of real environment.

Finally, the distribution process exports the interactive panoramic scenes to a series of different file formats, which provides the access to the interactive panoramic scenes in any computer or platform. Often, one or a series of software are required to open the created interactive panoramic scenes. This process is time consuming and asks users to operate several applications/software. However, it is possible to export the virtual simulation into HTML format, making it available through an online channel. A plethora of devices such as PCs, laptops, handheld devices, and head-mounted displays (HMD) are able to display the panoramas. Cloud technologies can be employed to make the interactive panorama accessible online which ultimately might facilitate real-time video feed and big data analysis.

\subsection{Hardware \& Software Requirements}

The creation of an interactive panorama requires specialized hardware and software. Due to the photo/videography nature of the process, cameras and camera lenses are the primordial hardware required. The two different capturing techniques involves very different equipment. The pivotal technique can employ any traditional camera, but in order to facilitate the capturing process, automated rotating platform, e.g. GigaPan Epic, Kolor Roundshot, Nodal Ninja, and spherical camera rigs, e.g. Pro6 Bullet360, Google Jump, Freedom360, have been developed. These systems often have a controller that allows users to take images at defined intervals to encompass the panorama, or manage multiple fixed cameras in prearranged angles to capture the 360-degree scene. These systems often yield image quality as good as the camera employed to take the photos or videos, but demand technical knowledge about photography to operate the devices and have a high cost entry point to provide high-quality imagery.

On the other hand, fish-eye lens or catadioptric lens systems can capture directly the panorama with little to no manipulation from the user. The camera is simply set at a location and captures the complete 360-degree scene with a single shot, often with dual fish-eye lens, like Ricoh Theta 15m, or with a single catadioptric lens, like V.360. Table 1 compares commercially available fish-eye lens cameras. These portable lightweight fisheye lens cameras are available at reasonable prices ( $\$ 170$ to $\$ 800$ ), provide sufficient photo/video graphic quality (Full HD to $4 \mathrm{~K}$ ), and also have the ability to record audio (microphones). 
Table 1. 360-degree Panoramic Fish-eye Lens Camera Comparison.

\begin{tabular}{cccccc}
\hline & $\begin{array}{c}\text { LG 360 } \\
\text { CAM }\end{array}$ & $\begin{array}{c}\text { Ricoh } \\
\text { Theta S }\end{array}$ & $\begin{array}{c}\text { Samsung } \\
\text { Gear 360 }\end{array}$ & $\begin{array}{c}\text { Nikon 360 } \\
\text { KeyMission }\end{array}$ & Bublcam \\
\hline Price & $\$ 170$ & $\$ 350$ & $\$ 350$ & $\$ 500$ & $\$ 800$ \\
Resolution & $2 \mathrm{~K}$ & Full HD & $4 \mathrm{~K}$ & $4 \mathrm{k}$ & Full HD \\
Lenses No. & 2 & 2 & 2 & 2 & 4 \\
Microphones No. & 3 & 2 & 2 & 2 & 1 \\
Weight & $97 \mathrm{~g}$ & $125 \mathrm{~g}$ & $152 \mathrm{~g}$ & $198 \mathrm{~g}$ & $280 \mathrm{~g}$ \\
\hline
\end{tabular}

Both techniques necessitate software to aid the visualization of the panorama. The pivotal technique uses stitching and editing software packages such as Panoweaver, Autopano, and PTGui, allowing the user to combine the image matrix and represent correct spherical coordinates. The projections on the surface of the sphere must exactly match the surrounding images, stitching the location of each source image on the corresponding section of the panorama. Conversely, the fish-eye technique usually requires stitching of minimum two images produced by the dual lens, which is mostly done internally by the camera's software.

Virtual interactive objects can be augmented into a panoramic scene to enhance immersion qualities. 2D and 3D augmentations are displayed into the panorama to provide data in form of texts, images, intersecting planes, geo-located maps, building floor plans, among many other possibilities. In order to make a scene navigable, controls in the form of graphical user interface (GUI) can be implemented. Other methods of navigation such as mouse drag-and-drop, game controllers (e.g. Xbox or Wii), or motion sensors (e.g., Oculus Touch, Leap Motion, or Kinect) are available to complement the GUIs.

\section{TECHNICAL DEVELOPMENT OF 360-DEGREE INTERACTIVE PANORAMAS USING A PILOT STUDY}

This pilot study shows how it is possible to create an interactive panoramic scene following the workflow described in this paper. The case study uses a construction site on the University of Florida Campus to create interactive panoramas and describes the creating and augmentation process of the interactive panorama. This test location is chosen as an example of a complex construction environment where it is constantly required to document the progress of the project. The ultimate goal is to use 360-degree interactive panoramas to not only create a virtual tour of such complex construction project but also use it as a documentation and asset management method. The outcome might be used to locate and visualize different building components, monitor, depict, and preserve conditions and document defective work or problem situations. The objective of this pilot study is to demonstrate the technical feasibility and practicality of creating interactive panoramic scenes of a real-world construction project using the described workflow. A series of locations and a path to navigate in the construction site was selected (points 1-3 in Figure 3) to provide a large amount of relevant visual information. Next, the sequential 360-degree images and videos were taken in the selected path locations. For this task, the dual fish-eye camera Ricoh Theta $S$ was used to collect the 
data in video and photo formats. Total of 42 pictures and 9 videos were captured, from which two photos and one video were designated to the locations in one presented path. The gathered data was processed to correct the geometric distortions and stitch, encode and edit the panoramic scenes.

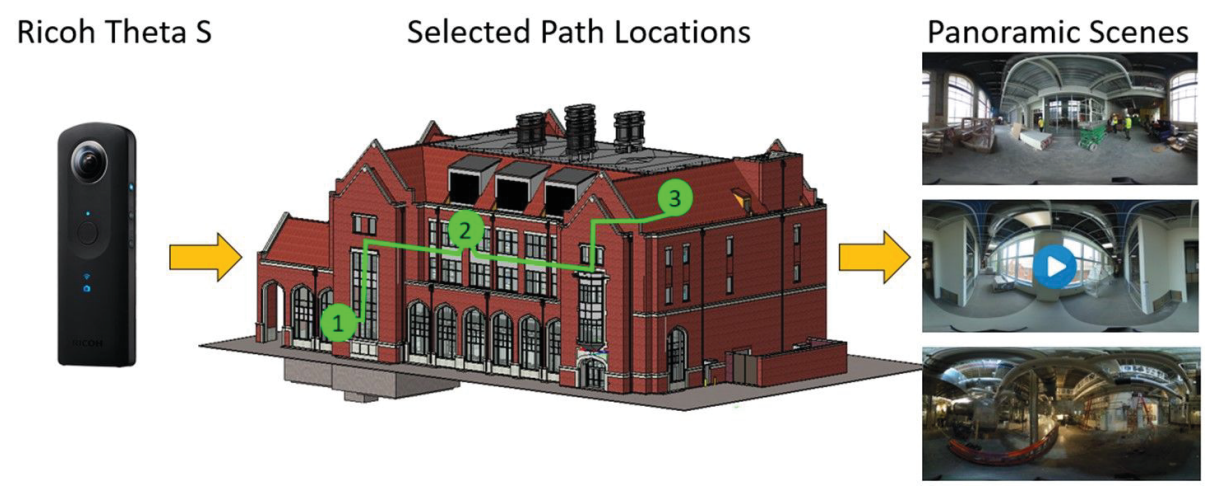

Figure 3. Capturing and Authoring Process.

For the immersion process, different layers of information were added to each panoramic scene using the Kolor Panotour software. Initially, each panoramic scene was introduced in the software package, arranged in the desired sequential order and linked together to provide a logical navigational sequence. Figure 4 illustrates the augmentation for the location's path, displaying the interconnections of the interactive panoramas and the sequence of navigation.

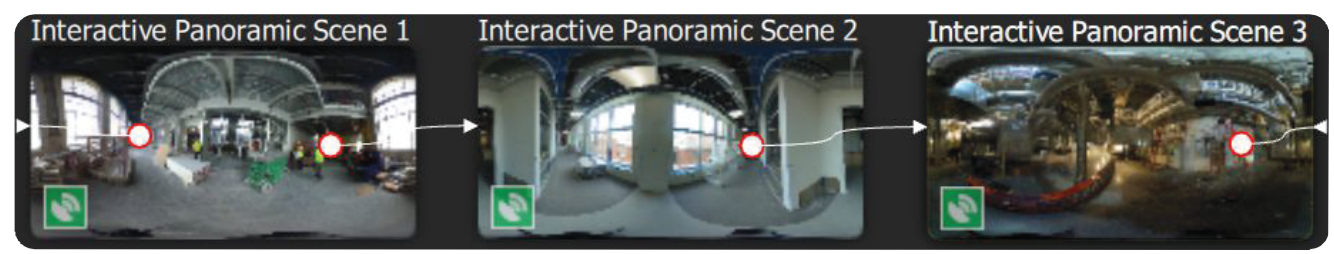

Figure 4. Immersion Process - Navigation.

Figure 5 demonstrates the augmentations used for this pilot study. Text tags to identify focus points, a title for the interactive panoramic scene, navigational arrows to enter and exit scenes, a geo-location of the panoramic scene (live google street map), the floor plan with the location of the user (JPEG image), a graphical user interface (GUI) to navigate the panoramic scenes, and a view of the computer generated building model for comparison (JPEG image) were implemented.

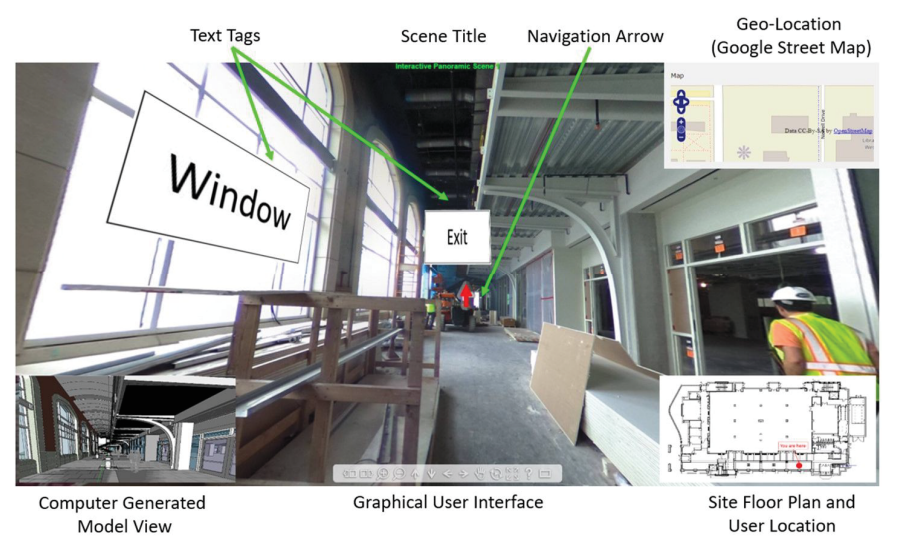

Figure 4. Immersion Process - Layers of Information 
Finally, in the distribution process, the interactive panoramic scenes were exported into HTML format, allowing any platform to view it. Figure 6 shows the finished interactive panoramic scene as it is displayed in HTML format on a tablet. The navigation GUI would also allow users to zoom in and out of the scene to explore in detail the augmentations. The texts, geo-location, floor plan, and model view are all visible to the user in each interactive panoramic scene.

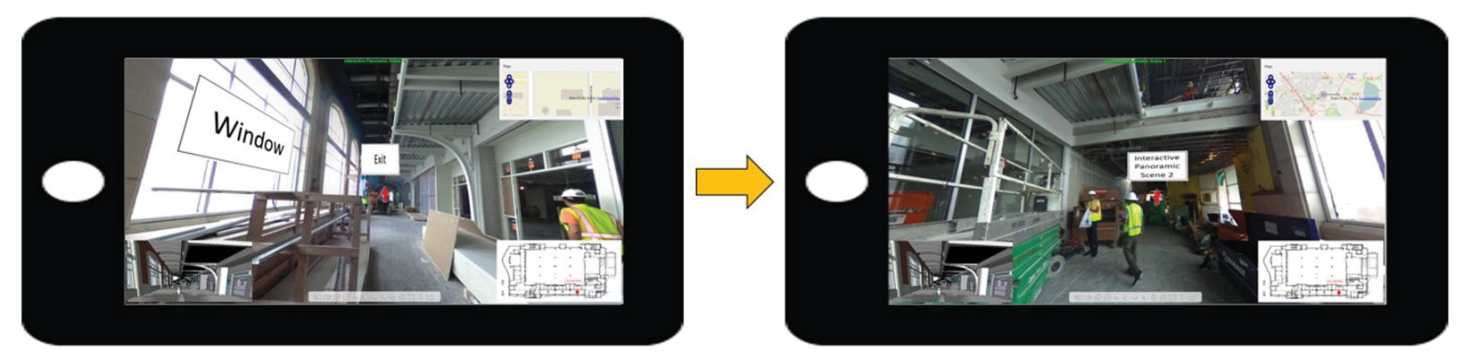

Figure 6. Distribution Process - An Interactive Panoramic Scene

\section{SUMMARY AND FURTHER STUDY}

Interactive panoramic scenes are low-cost, high-quality, immersive virtual reality that captures real environments using uninterrupted images or videos, augmented with realworld data. This platform offers a suitable method to visualize complex construction projects. It has been found that similar techniques have been used previously in the construction literature, but ubiquitous technologies have simplified the process down to a handheld camera or smartphone that acquires pictures in a few seconds with just a click. Most of the authoring related processes has been automated to visualize the panoramas and has allowed uncomplicated navigation through the scenes. A plethora of humancomputer interfaces are available to interact with the panoramic scenes, but HMDs present a very attractive platform with the inclusion of sound to enhance the user's immersion.

In the pilot study, photography and videography data was collected from a construction site to create a navigable series of panoramic scenes. A set of layers of information was added to the panoramic scenes to augment the information displayed, and then the panoramic scenes were exported to a PC and also HTML to ease its worldwide access. Further research contemplates the utilization of the workflow presented in this study for a larger scale project with the objective of documenting all construction stages throughout the project. It is recommended to test with real users the data layers implemented into the simulation to identify the ideal set of displayed information required in a panorama for construction-related applications. Additionally, a comparison should be performed regarding traditional virtual reality methods with interactive panoramas to clearly recognize practical advantages and limitations of both approaches in construction projects.

\section{REFERENCES}

Bourke, P. (2014). The Panorama: Applications to Science and Heritage Visualization. Lawrence Wilson Art Gallery, Web. <http://paulbourke.net/papers/lawrencewilson/>.

Côté S. (2011a). Augmented Reality for Infrastructure: A First Step. Published electronically on BE Communities. 
Côté S. (2011b). Augmented Reality for Underground Infrastructure: The Problem of Spatial Perception. Published electronically on BE Communities.

C. Brauer-Burchardt, and K. Voss (2001). A New Algorithm to Correct Fish-eye and Strong Wide-angle-lens-distortion from Single Images. Proceedings of 2001 International Conference on Image Processing, Vol.1, pp.225 - 228.

Dickinson, I., Riley, A., Tennant, J., and Ellis, R.C.T. (2004). Virtualsite: Photo-realism in the Classroom. Proceedings of COBRA, Leeds Metropolitan University.

Finch, E., and Wing, R. D. (1996). A Navigable Walkthrough Simulator for Built Environment Education: Archiwalk. Facilities, 14(10/11), 17-24.

Gheisari, M., Sehat N., and Williams G. (2015): Using Augmented Panoramic Views as an Online Course Delivery Mechanism in MOOCs. Proceedings of the $51^{\text {st }}$ ASC Annual International Conference.

Gheisari, M., Sabzevar, M.F., Chen, P., and Irizzary, J. (2016). Integrating BIM and Panorama to Create a Semi-Augmented-Reality Experience of a Construction Site. International Journal of Construction Education and Research, 303-316.

Gheisari, M., Sabzevar, M.F., Chen, P., and Irizzary, J. (2016). An Augmented Panoramic Environment to Access Building Information on a Construction Site. Proceedings of the $52^{\text {nd }}$ ASC Annual International Conference.

Mei, Q., and Wing, R. (1999). Robotic 360o Photography for Virtual Site Visits. IEEE International Conference on Information Visualization, 214-219.

Perez-Ramos, A. (2016). NCTech Application Notes \& Case Studies. Retrieved from: https://www.nctechimaging.com/ (Accessed on November 12, 2016).

Nayar S.K. (1997). Omnidirectional Vision. The Eighth International Symposium of Robotics Research, Hayama, Japan.

Nayar S.K., and Boult, T. (1998). Omnidirectional Vision Systems: Report. Department of Computer Science Department of EE and CS Columbia University Lehigh University New York, N.Y. 10027 Bethlehem, PA 18015

Vanijja, V., and Horiguchi, S. (2001) 360 Interactive Video Scenes with Multi-Directional Moving Capability. Multidirectional Moving Capability”. Proceedings of ICAT2001, pp.209-212.

Vanijja, V., and Horiguchi, S. (2006). A Stereoscopic Image-Based Approach to Virtual Environment Navigation. The Computer, the Internet and Management, 14, pp. 6881.

Wither J., Tsai W-T., and Azuma R. (2011). Indirect Augmented Reality. Computers \& Graphics, 35, pp. 810-822. 